\title{
Polarization dependent intra-band dynamics in semiconductor optical amplifiers
}

\author{
Severine Philippe ${ }^{1 *}$, Frederic Surre ${ }^{1,2}$, A. Louise Bradley ${ }^{1}$, Ramon Maldonado-Basilio ${ }^{2,3}$, Brendan \\ Kennedy ${ }^{2,4}$, Pascal Landais ${ }^{2}$, Member IEEE, Horacio Soto-Ortiz ${ }^{3}$, \\ ${ }^{1}$ School of Physics, Trinity College Dublin, Dublin 2, Ireland, \\ ${ }^{2}$ School of Electronics Engineering, Dublin City University, Glasnevin, Dublin 9, Ireland. \\ ${ }^{3}$ CICESE Research Center, Applied Physics Division, Carretera Tijuna-Ensenada, Ensenada 22860, Baja \\ California, Mexico. \\ ${ }^{4}$ Electrical Engineering Department, Universidad de Santiago de Chile, Avda. Ecuador 3519, Estacion Central, \\ Santiago, Chile. \\ * Tel: (353) 1896 2677, Fax: (353) 1671 1759, e-mail: philipps@tcd.ie
}

\begin{abstract}
The polarization dependence of gain dynamics in a bulk semiconductor optical amplifier has been investigated using a 2.5 ps pump-probe set-up in a counter-propagation configuration. A theoretical model based on the carrier density rate equation has been developed to simulate the experiment. Comparison of experimental and computational results highlights the dependence of the intra-band dynamics on both the energy of the injected pump pulse and the polarization orientation of the pump and probe signals. The power dependence of the saturation energy and gain compression factor are extracted and seen to be a function of polarization.
\end{abstract}

Keywords: Semiconductor optical amplifiers, intraband effects, pump-probe measurements, counter-propagation

\section{INTRODUCTION}

The polarization dependence of Semiconductor Optical Amplifiers (SOA) is one major concern for telecommunication applications. Ways to design polarization insensitive SOA have been proposed. While encouraging results have been obtained under the injection of $\mathrm{CW}$ signals, it cannot be assumed that the dynamic response of the SOA will also be polarization insensitive.

In order to address this question, the polarization resolved gain dynamics have been investigated using a picosecond pump-probe technique. To corroborate and further elucidate the experimental results a phenomenological model based on the carrier density rate equation has been developed. Since the pulse width of the propagating pump and probe signals is smaller than the carrier recovery time, the set of equations governing the general pulse propagation can be simplified according to some assumptions detailed in [1] and [2] and analytically solved. It features a phenomenological correction factor able to describe the non-linear phenomena of carrier heating $(\mathrm{CH})$ and spectral hole burning (SHB) [3] and [4].

\section{PUMP-PROBE SET-UP: EXPERIMENTAL RESULTS}

The device under test is a commercial $1.5 \mathrm{~mm}$ long bulk InGaAsP/InP traveling wave SOA. It is biased at $350 \mathrm{~mA}$ and temperature regulated at $20^{\circ} \mathrm{C}$ by means of a Peltier cooler. Under these experimental conditions the gain peak is at $1580 \mathrm{~nm}$ and the material gain in TM is slightly larger than in TE. More information on the device can be found in [5]. The experimental set-up is a pump-probe counter-propagation experiment and is shown in Fig.1. The $1580 \mathrm{~nm}$ pulsed input is produced using a fibre based femtosecond laser, with a repetition rate of 82 MHz. The pulse width after dispersion in the fiberised output is $2.5 \mathrm{ps}$ and a grating filter with a $1 \mathrm{~nm}$ spectral width is used to select the wavelength. The pulses are coherent and one is delayed with respect to the other using a variable delay stage with a time increment of 530fs. The pulses propagate in free space, allowing for complete control and preservation of the state of polarization at both the input and the output of the device The polarization of each beam is controlled independently with a quarter wave plate (QWP) and a half wave plate (HWP). The light is coupled in and out of the SOA using two antireflection coated aspheric lenses. An interference filter is used to isolate the $1580 \mathrm{~nm}$ probe signal from the broad amplified spontaneous emission (ASE) and the signal is detected using a fast infrared photodiode and a lock-in technique.

As shown Fig. 2, different gain compression behaviour is observed for each pump probe polarization combination. The discrepancy between the TM mode gain compression and the TE mode gain compression is most likely due to strain effects [6] and [7]. Commonly present in bulk devices, even for a negligible lattice mismatch [6] and strain has the consequence of removing the degeneracy of the light and heavy hole valence

bands. The transitions associated with TE and TM modes are different with TM transitions involving light holes while TE transitions involve a mixture of light (25\%) and heavy (75\%) holes [7]. As a consequence while polarization combinations, show an increase in gain compression with increasing pump pulse energy, the amount 
of gain compression differs due to the different transitions involved for each eigen mode from the conduction band to the light and heavy holes valence bands. In the co-polarized cases, the pump signal depletes the carriers by the same transition than that used for probe amplification. The slow recovery component is much larger in the case of TM mode gain compression than for the TE mode. Also the pump energy required to reach the same level of gain compression is lower for TM than for TE which is consistent with the carrier states density of the heavy holes valence band (TE) being higher than that of the light holes (TM).

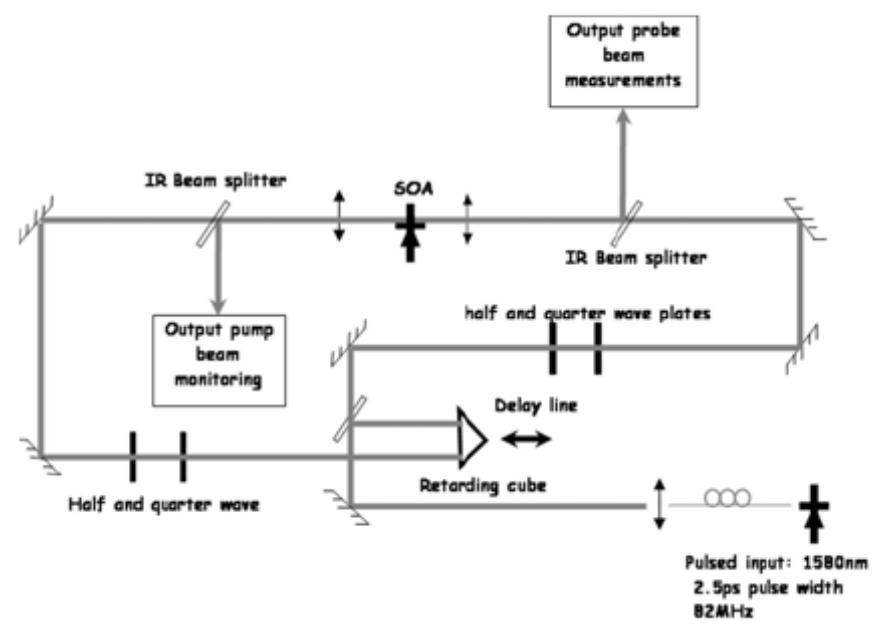

Figure 1.Pump-probe counter-propagation experimental set-up.

In the cross-polarized cases, a larger cross-saturation is observed for the TE polarized pump and TM polarized probe case. As the TE transitions involve of $25 \%$ light holes, this can lead to a significant reduction of light hole population at high pump powers for a TE polarized pump, and thus a decrease in the TM probe gain. On the other hand the decrease in light hole concentration due to a TM pump will only concern at most $25 \%$ of the holes associated with the TE probe transitions.

\section{PUMP-PROBE SET-UP: MODEL}

We have developed a simulation of the experiment based on [1] and [2]. It is based on the integration of the first order time derivation of the carrier density given by:

$$
\frac{d N}{d t}=\frac{I q}{V}-\frac{N}{\tau_{c}}-\frac{a\left(N-N_{0}\right)}{\hbar \omega_{0}}|E|^{2}
$$

where $\mathrm{N}$ is the carrier density, I the injection current, $\theta$ the electron charge, $\varsigma$ the active volume, $\tau \chi$ the spontaneous carrier lifetime, \}wo the photon energy, $\alpha$ the differential gain taking into account intra-band effects, and No the carrier density at transparency. The integration takes into account the time variation of electric fields of both the pump and the probe, summarized as E in Eq. (1).

In order to take into account the intra-band effects, which are relevant for picosecond pulse propagation in the SOA, the gain material, $g$, is modified following the formula:

$$
\widetilde{g}(t)=\frac{g(t)}{f(t)}
$$

where $\widetilde{g}$ is the modified gain and $\mathrm{f}$ is the correction factor which can be expressed by

$$
f(t)=1+\varepsilon S(t)=1+\varepsilon \frac{2 \varepsilon_{o p} \eta \eta_{g}|A(t)|^{2}}{\hbar \omega_{0}}
$$

where $\varepsilon$ is the non-linear gain compression factor, $\mathrm{S}$ is the total photon density generated by the saturating signal (the pump pulse), $\varepsilon_{o p}$ is the vacuum permittivity, $\mathrm{A}$ is the slow varying envelope associated with the optical pulse, and $\eta$ is the refractive index, $\eta_{\mathrm{g}}$ the group index. 
TETE

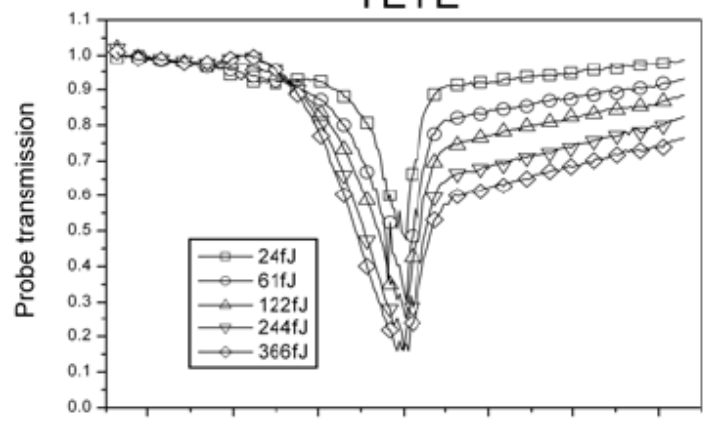

TMTE

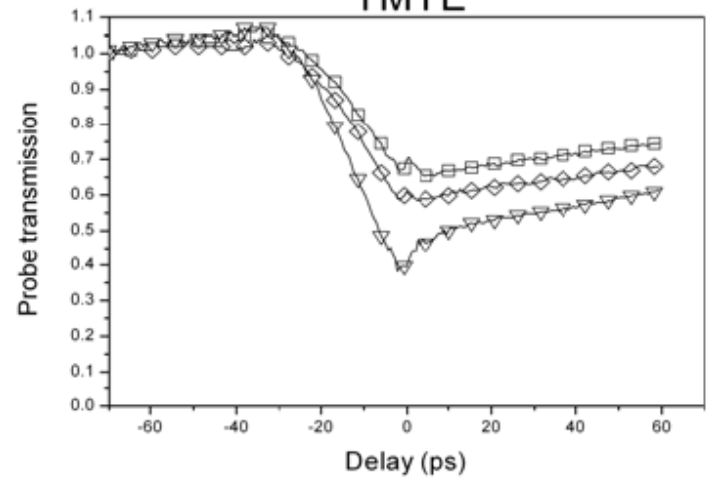

TMTM

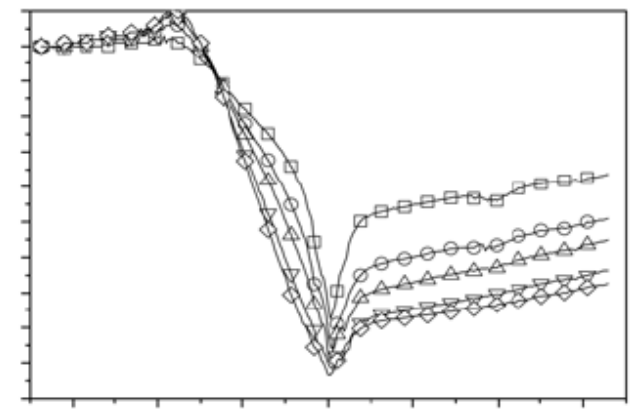

TETM

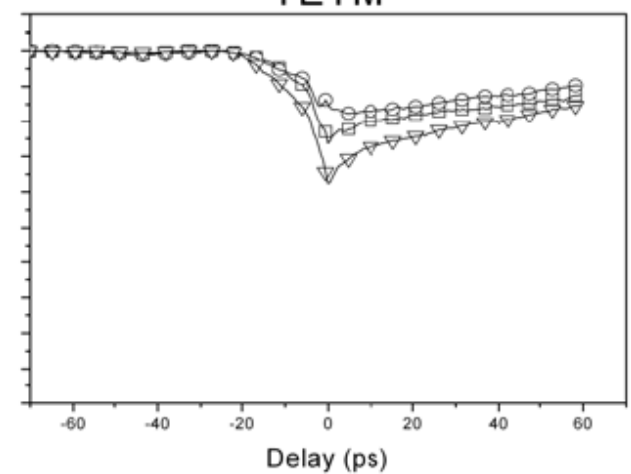

Figure 2. Experimental transmission of the probe signal in co- and cross-polarized cases for 24fJ, 61fJ, 122fJ, $244 \mathrm{fJ}$ and $366 \mathrm{fJ}$ values of pump pulse energies. The probe energy is set at $12 \mathrm{fJ}$.

TETE

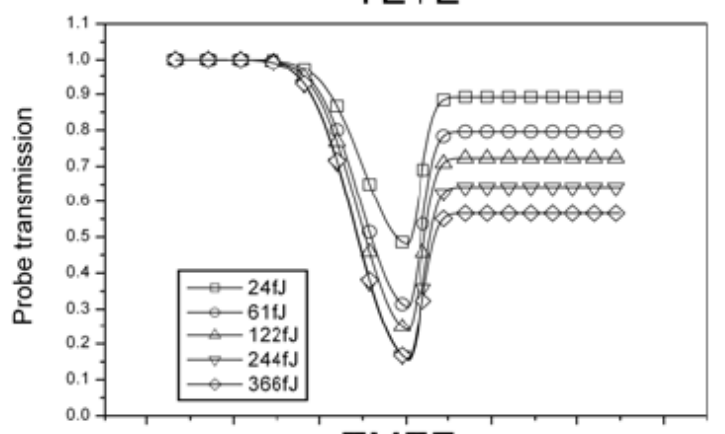

TMTE

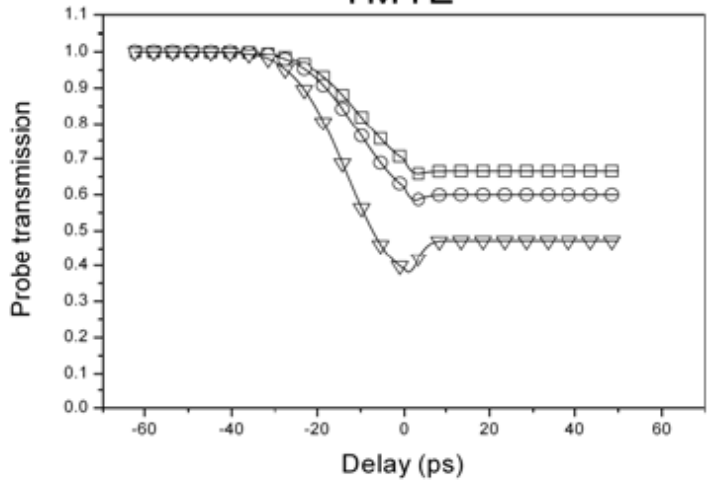

TMTM

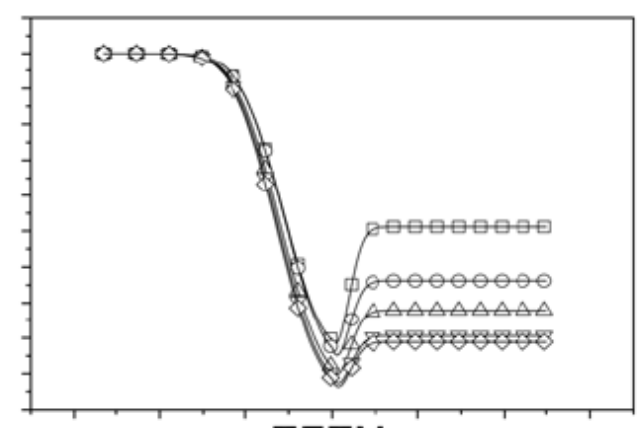

TETM

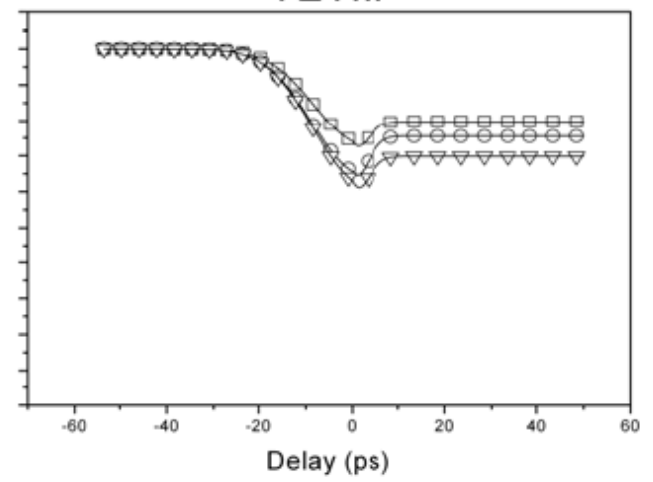

Figure 3. Simulated transmission of the probe signal in co- and cross-polarized cases for pump pulse energies of $24 \mathrm{fJ}, 61 \mathrm{fJ}, 122 \mathrm{fJ}, 244 \mathrm{fJ}$ and $366 \mathrm{fJ}$. The probe signal energy is $12 \mathrm{fJ}$. 
In the co-polarized case, the same correction factor and the same saturation energy are used for both pump and probe signals. For the cross-polarized case, as the pump and probe pulses are propagating along different waveguide eigen-axes, the gain model assumes slightly different gain parameters, namely for correction factor and saturation energy. It is also assumed that the pump pulse induces the amplifier saturation, which will be of different strength for each of the two waveguide eigen-axes based on the experimentally observed probe transmission curves, shown in Fig. 2. More information on this model can be found in [8]. As can be seen in Fig.3 the simulated results present strong similarities with those experimental results shown in Fig. 2. This is achieved by taking into account the dependence of the energy saturation and the gain compression on the polarization state of the pump and probe.

\section{CONCLUSIONS}

Pump-probe studies using 2.5 ps pulses in a contra-propagation set-up clearly show the polarization and power dependence of the gain compression. A model based on traveling waves in SOA has been developed to simulate the experiment taking into account the gain saturation by introducing the energy saturation and a compression factor. Excellent agreement between the experimental results and the simulation has been obtained demonstrating a polarization dependence of the intraband gain dynamics.

\section{ACKNOWLEDGEMENTS}

This research has been supported by Enterprise Ireland under the Commercialisation Fund Technology

Development programme, project number CFTD/06/IT/332.

\section{REFERENCES}

[1] G.P.Agrawal, N.A.Olsson, "Self-pulsating modulation and spectral broadening of optical pulses in semiconductor laser amplifiers", IEEE J. Quant. Electr., vol 25, Nov. 1989

[2] N. A. Olsson and G. P. Agrawal. "Spectral shift and distortion due to self-phase modulation of picosecond pulses in $1.5 \mu \mathrm{m}$ optical amplifiers", Appl. Phys. Lett., vol. 55. Jan. 1989.

[3] M. Y. Hong, Y. H. Chang, A. Dienes, J. P. Heritage and P. J. Delfyett. "Subpicosecond pulse amplification in semiconductor laser amplifiers: theory and experiment" IEEE J. of Quant. Electr., vol. 30, Apr 1994.

[4] M. Y. Hong, Y. H. Chang, A. Dienes, J. P. Heritage, P. J. Delfyett, S. Dijaili and F. G. Patterson. "Femtosecond self- and cross-phase modulation in semiconductor laser amplifiers" IEEE J. of Selected Topics in Quant. Electron.. Vol. 2, No. 3. 1996.

[5] R. P. Schreieck, M. H. Kwakernaak, H. Jäckel, and H. Melchior, "All-optical switching at multi-100-Gb/s data rates with Mach-Zehnder interferometer switches" IEEE J. of Quant. Electron., vol. 33, Aug. 2002.

[6] T. D. Visser, H. Blok, B. Demeulenaere, and D. Lenstra, "Confinement factors and gain in optical amplifiers," IEEE J. of Quantum Electron., vol. QE-33, pp. 1763-1766, 1997.

[7] E. P. O’Reilly and A. R. Adams, "Band-structure engineering in strained semiconductor lasers," IEEE J. Quant. Electron., vol. 30, pp. 366-379, 1994.

[8] S.Philippe, A.L.Bradley, F.Surre, P.Landais, R.Maldonado-Basilio, H.Soto-Ortiz, B.F.Kennedy, "Time resolved polarization dependence of intra-band effects in semiconductor optical amplifier", submitted to IEEE J. Quant. Electron., April 2007. 